\title{
Synthesis of general linear systems with repeated filtering in consecutive fractional Fourier domains
}

\author{
M. Fatih Erden and Haldun M. Ozaktas \\ Department of Electrical Engineering, Bilkent University, 06533 Bilkent, Ankara, Turkey
}

\author{
Received September 4, 1997; revised manuscript received January 22, 1998; accepted January 23, 1998
}

\begin{abstract}
The optical and digital implementations of general linear systems are costly. Through several examples we show that either exact realizations or useful approximations of these systems may be implemented in the form of repeated-filtering operations in consecutive fractional Fourier domains. These implementations are much cheaper than direct implementations of general linear systems. Thus we may significantly decrease the implementation costs of general linear systems with little or no decrease in performance by synthesizing them with the proposed repeated-filtering method. (C) 1998 Optical Society of America [S0740-3232(98)01706-2]

OCIS code: 070.2580 .
\end{abstract}

\section{INTRODUCTION}

Space and spatial-frequency domains are the special cases of so-called fractional Fourier domains. They correspond to the zeroth and first fractional Fourier domains, respectively. Thus filtering in the space domain is equivalent to filtering in the zeroth fractional domain. Likewise, filtering in the spatial-frequency domain is equivalent to filtering in the first fractional domain. In Refs. 1-4 it is shown that the added degree of freedom afforded by the order parameter $a$ allows improved performance in a variety of signal processing applications. Furthermore, since both the digital ${ }^{5}$ and optical ${ }^{6-9}$ implementations of the fractional Fourier transformation do not lead to extra work compared with the conventional Fourier transformation, these improvements come at no cost. Two important applications of filtering in a single fractional Fourier domain are discussed in Refs. 3 and 4. In Ref. 3 optimal Wiener filtering has been generalized to fractional Fourier domains, and in Ref. 4 desired mutual intensity functions for a given input mutual intensity have been synthesized by filtering in fractional Fourier domains. Later, in Ref. 10, we generalized singlefractional-Fourier-domain filtering to repeated filtering in consecutive fractional Fourier domains and showed that we may obtain a considerable improvement in signal restoration compared with that for single-domain filtering. In Ref. 10 we also compared the repeated-filtering method with the optimum linear estimation method in signal restoration and saw that use of the repeated-filtering method may result in significant computational savings with little or no sacrifice in performance.

Thus, in Ref. 10, repeated filtering in consecutive fractional Fourier domains has been successfully applied to signal restoration. In this paper we apply the repeatedfiltering method for the synthesis of general linear systems. In other words, we synthesize the linear systems by introducing several multiplicative filters at different consecutive fractional Fourier domains. The configura- tion is shown in Fig. 1. Here we apply the first filter in the zeroth fractional domain (the space domain), the second filter in the $a_{1}$ th fractional domain, the third filter in the $\left(a_{1}+a_{2}\right)$ th fractional domain, and so on. Our aim is to find the optimal filter profiles in the repeated-filtering configuration in order to approximate a given linear system.

To implement the configuration in Fig. 1 both digitally and optically, we assume that the input and output signals are represented by one-dimensional arrays of size $N$. These arrays may also represent $\sqrt{N} \times \sqrt{N}$ images.

Any linear system can be implemented optically with conventional approaches such as matrix-vectormultiplier architectures ${ }^{11}$ or multifacet architectures, ${ }^{12}$ but these are not space-bandwidth efficient. ${ }^{13}$ In other words, to implement a given general linear transformation represented by an $N \times N$ matrix, it is necessary to employ an optical system whose space-bandwidth product is $N^{2}$. However, the proposed repeated-filtering configuration provides a space-bandwidth-efficient method in the sense that the space-bandwidth product of the optical system need be only of the order of $N$. Let us illustrate the concept of space-bandwidth efficiency with an example. Assume that the maximum space-bandwidth product of the optical elements that we are allowed to use is 10,000. With these optical elements, when we apply the inefficient conventional approaches, we can synthesize only the linear systems between input and output arrays of size 100 (or $10 \times 10$ images). However, again with the same optical elements, when we apply our repeated-filtering method, we can this time synthesize the linear systems between input and output arrays of size 10,000 (or $100 \times 100$ images). The optical implementation cost of our space-bandwidth-efficient repeated-filtering configuration depends highly on the number of stages used. Here we desire to reduce the cost by synthesizing linear transformation kernels with the use of a moderate number of filters in the repeated- 


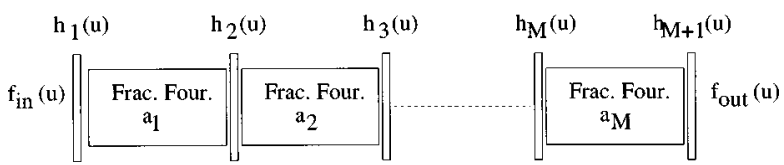

Fig. 1. Configuration for repeated filtering in consecutive fractional Fourier domains.

filtering configuration. As phase-only filters are sometimes preferred to those with arbitrary complex amplitudes, we also consider the repeated-filtering problem under this constraint.

In addition to their optical implementations, digital implementations of general linear systems are also costly. To implement a general linear transformation represented by an $N \times N$ matrix, we need $O\left(N^{2}\right)$ computation time. However, the digital implementation of our repeated-filtering configuration requires $O(M N \log N)$ computation time (where $M$ is the number of stages sandwiched between the filters). Thus our digital implementation cost also depends on the number of stages, and we can again reduce the cost if we are able to obtain a good approximation of the given linear system by using a moderate number of filters.

We start the paper by giving a brief introduction to fractional Fourier transformation in Section 2. Then, in Section 3, we define the problem of approximating linear systems by using repeated filtering in consecutive domains and show that it can be reduced to a simple canonical form. In this section we also propose an iterative algorithm to obtain the filter profiles in the repeatedfiltering configuration necessary for the synthesis of a desired linear system. Then we modify the iterative algorithm for phase-only filters. Last, in Section 4, we discuss the applications of the repeated-filtering method through several examples.

\section{FRACTIONAL FOURIER TRANSFORMATION}

The $a$ th-order fractional Fourier transformation $p_{a}(u)$ of $p(u)$ is defined for $0<|a|<2$ as

$$
\begin{aligned}
p_{a}(u)= & (1-j \cot \phi)^{1 / 2} \int_{-\infty}^{\infty} \exp \left[j \pi \left(u^{2} \cot \phi\right.\right. \\
& \left.\left.-2 u u^{\prime} \csc \phi+u^{\prime 2} \cot \phi\right)\right] p\left(u^{\prime}\right) \mathrm{d} u^{\prime},
\end{aligned}
$$

where $\phi=a \pi / 2$. The kernel is defined separately for $a$ $=0 \quad$ and $\quad a= \pm 2 \quad$ as $\quad B_{0}\left(u, u^{\prime}\right) \equiv \delta\left(u-u^{\prime}\right)$ and $B_{ \pm 2}\left(u, u^{\prime}\right) \equiv \delta\left(u+u^{\prime}\right)$, respectively. ${ }^{14}$ The definition is easily extended outside the interval $[-2,2]$ through $\mathscr{F}^{4 i+a} \hat{q}=\mathscr{F}^{a} \hat{q}$ for any integer $i$. Both $u$ and $u^{\prime}$ are dimensionless variables.

Some essential properties of the transformation are (1) it is linear, (2) the first-order transformation $(a=1)$ corresponds to the common Fourier transformation, and (3) it is additive in index $\left(\mathscr{F}^{a_{1}} \mathscr{F}^{a} \hat{q}=\mathscr{F}^{a_{1}+a_{2}} \hat{q}\right)$. Other properties may be found in Refs. 1, 6, 7, and 14-17.

The fractional Fourier transformation kernel in Eq. (1) has only a single free parameter, its fraction. By allowing for the possibility of a residual quadratic-phase term and a scale factor, we can generalize this kernel as ${ }^{8,18}$

$$
\begin{aligned}
h\left(x, x^{\prime}\right)= & K \exp \left(j \pi x^{2} / \lambda R\right) \exp \left[\frac { j \pi } { s ^ { 2 } } \left(\frac{x^{2}}{M^{2}} \cot \phi\right.\right. \\
& \left.\left.-2 \frac{x x^{\prime}}{M} \csc \phi+x^{\prime 2} \cot \phi\right)\right],
\end{aligned}
$$

where $\phi=a \pi / 2$. This kernel maps a function $p(x / s)$ into $K^{\prime} \exp \left(j \pi x^{2} / \lambda R\right) p_{a}(x / s M)$, where $p_{a}(\cdot)$ is the $a$ th-order fractional Fourier transformation of $p(\cdot)$ and $K^{\prime}$ is a new constant. In Eq. (2) $s$ is the unit in which $x$ and $x^{\prime}$ are measured, $M>0$ is referred to as the scale factor associated with the transformation, and $R$ is the radius of the spherical surface on which the scaled fractional Fourier transformation is observed. We see from Eq. (2) that the pure mathematical form of the fractional Fourier transformation in Eq. (1) is obtained when $s$ $=1, M=1$, and $R=\infty$.

Axially symmetric quadratic-phase optical systems under the standard approximations of Fourier optics ${ }^{19}$ are closely related to the fractional Fourier transformation. 8 Thin lenses, arbitrary sections of free space (under the Fresnel approximation), quadratic graded-index media, and any combinations of these belong to the class of quadratic-phase systems. We characterize the members of quadratic-phase systems through $^{8,20-23}$

$$
\begin{aligned}
p_{\text {out }}(x) & =\int_{-\infty}^{\infty} Q\left(x, x^{\prime}\right) p_{\text {in }}\left(x^{\prime}\right) \mathrm{d} x^{\prime}, \\
Q\left(x, x^{\prime}\right) & =K_{q} \exp \left[\frac{j \pi}{s^{2}}\left(\alpha x^{2}-2 \beta x x^{\prime}+\gamma x^{\prime 2}\right)\right],
\end{aligned}
$$

where $K_{q}$ is a complex constant and $\alpha, \beta$, and $\gamma$ are real constants. Thus, apart from the constant factor $K_{q}$, which has no effect on the resulting spatial distribution, a member of the class of quadratic-phase systems is completely specified by the three parameters $\alpha, \beta$, and $\gamma$. We deduce the close relationship between the quadraticphase systems and the fractional Fourier transformation by comparing the kernels in Eqs. (2) and (3b). By setting $\alpha=(\cot \phi) / M^{2}+1 / \lambda R, \beta=(\csc \phi) / M$, and $\gamma=\cot \phi$, we see that these two kernels are equivalent. Thus we conclude that any quadratic-phase system can be interpreted as a fractional Fourier transformer.

Fractional Fourier transformation can be easily realized optically in the same manner as the ordinary Fourier transformation, ${ }^{6-9,24,25}$ which has led to many applications in optical signal processing. ${ }^{6-9,15,16,24,26,27}$ In Ref. 5 a fast algorithm that calculates the fractional Fourier transformation in $O(N \log N)$ time is also presented. As this transformation is a generalization of the common Fourier transformation, it becomes a natural candidate for improving the results in applications in which the Fourier transformation is widely used. Some of these applications are space-variant filtering and signal detection, ${ }^{1-3,28,29}$ time-variant or space-variant multiplex- 
ing and data compression, ${ }^{1}$ correlation, matched filtering, and pattern recognition, ${ }^{30,31}$ signal synthesis, ${ }^{32}$ radar, ${ }^{3}$ generalization of Wiener filtering to fractional Fourier domains, $^{2,3,33}$ and phase retrieval. ${ }^{34}$

\section{SYNTHESIS OF A DESIRED LINEAR TRANSFORMATION KERNEL}

We characterize one-dimensional linear systems through

$$
f_{\text {out }}(u)=\int T_{d}\left(u, u^{\prime}\right) f_{\text {in }}\left(u^{\prime}\right) \mathrm{d} u^{\prime},
$$

and, similarly, two-dimensional ones through

$$
f_{\text {out }}(u, v)=\iint T_{d}\left(u, v, u^{\prime}, v^{\prime}\right) f_{\text {in }}\left(u^{\prime}, v^{\prime}\right) \mathrm{d} u^{\prime} \mathrm{d} v^{\prime},
$$

where $T_{d}\left(u, u^{\prime}\right)$ and $T_{d}\left(u, v, u^{\prime}, v^{\prime}\right)$ are the kernels of the one-dimensional and two-dimensional linear systems, respectively. We see from these equations that linear systems are fully characterized by their associated transformation kernels. Thus we will define our problem of linear system synthesis as the synthesis of their associated transformation kernels. We will then show that the problem reduces to a simple canonical form and also introduce the discrete version of this canonical form. Then we will propose a method for the solution of the problem. At the end of this section, we will also consider the repeated-filtering problem with phase-only filters.

\section{A. Mathematical Definition of the Problem}

We will first restrict ourselves to one-dimensional systems. The basic filtering configuration has already been depicted in Fig. 1. As the transformation stages in this figure, we will consider the more general three-parameter definition given in Eq. (2) or, equivalently, the quadraticphase system defined in Eqs. (3). Filtering in a single quadratic-phase system domain was discussed in Ref. 35.

Let there be $M$ consecutive quadratic-phase systems. We place $M+1$ filters such that each quadratic-phase system becomes sandwiched between two filters. The configuration is shown in Fig. 2(a). In this figure $h_{1}(u), h_{2}(u), \ldots, h_{M+1}(u)$ are the filter functions, and $Q_{l}\left(u, u^{\prime}\right), l=1, \ldots, M$, are the quadratic-phase system

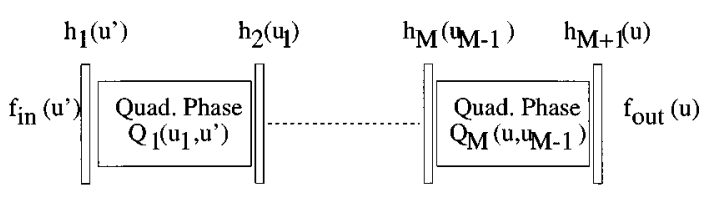

(a)

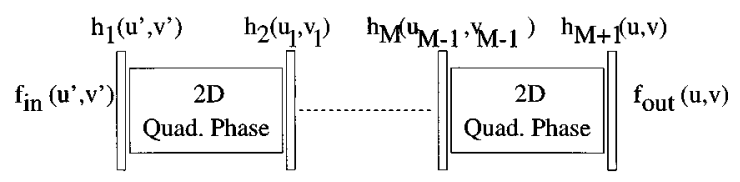

(b)

Fig. 2. Repeated filtering in consecutive (a) one-dimensional (1D) quadratic-phase systems, (b) two-dimensional (2D) quadratic-phase systems. kernels defined in Eq. (3b). [Here we scale the variables of all $Q_{l}\left(x, x^{\prime}\right), l=1, \ldots, M$, with $s$. That is, we replace $x / s$ with $u$ and $x^{\prime} / s$ with $u^{\prime}$. Now $u$ and $u^{\prime}$ are dimensionless variables.] In such a configuration $f_{\text {out }}(u)$ is related to $f_{\text {in }}\left(u^{\prime}\right)$ through a linear transformation [as in Eq. (4)] whose kernel is expressed as

$$
\begin{aligned}
T\left(u, u^{\prime}\right)= & \iint \cdots \int \mathrm{d} u_{1} \cdots \mathrm{d} u_{M-1} h_{1}\left(u^{\prime}\right) \\
& \times h_{2}\left(u_{1}\right) \cdots h_{M}\left(u_{M-1}\right) h_{M+1}(u) \\
& \times Q_{M}\left(u, u_{M-1}\right) Q_{M-1}\left(u_{M-1},\right. \\
& \left.u_{M-2}\right) \cdots Q_{1}\left(u_{1}, u^{\prime}\right) .
\end{aligned}
$$

As can be seen, $T\left(u, u^{\prime}\right)$ has a specific form. We want to choose the filters $h_{1}(u), h_{2}(u), \ldots, h_{M+1}(u)$ and the quadratic-phase system parameters $\alpha_{1}, \beta_{1}, \gamma_{1}, \ldots, \alpha_{M}$, $\beta_{M}, \gamma_{M}$ such that $T\left(u, u^{\prime}\right)$ is as close as possible to the desired kernel $T_{d}\left(u, u^{\prime}\right)$. Quantitatively, we will try to minimize the mean-square error (MSE), defined as

$$
e=\iint\left|T_{d}\left(u, u^{\prime}\right)-T\left(u, u^{\prime}\right)\right|^{2} \mathrm{~d} u \mathrm{~d} u^{\prime} .
$$

Let us now define our repeated-filtering problem in two dimensions. The fractional Fourier transformation can be generalized to two dimensions in two ways. One of them is the separable two-dimensional fractional Fourier transformation, and its kernel can be easily formed as the multiplication of two one-dimensional fractional Fourier transformation kernels. The other definition of the twodimensional fractional Fourier transformation has a nonseparable form, which has been defined in Refs. 36 and 37. However, the form of the most general twodimensional quadratic-phase system includes both cases. Thus we will consider such general quadratic-phase systems, whose kernel is given by

$$
\begin{aligned}
Q_{k}\left(x, y, x^{\prime}, y^{\prime}\right)= & K_{k} \exp \left[\frac { j \pi } { s ^ { 2 } } \left(a_{k} x^{2}+b_{k} y^{2}+c_{k} x^{\prime 2}\right.\right. \\
& \left.\left.+d_{k} y^{\prime 2}+2 e_{k} x y+2 f_{k} x x^{\prime}\right)\right] \\
& \times \exp \left[\frac { j \pi } { s ^ { 2 } } \left(2 g_{k} x y^{\prime}+2 h_{k} y x^{\prime}\right.\right. \\
& \left.\left.+2 m_{k} y y^{\prime}+2 n_{k} x^{\prime} y^{\prime}\right)\right]
\end{aligned}
$$

where $K_{k}$ is complex and all the remaining parameters (i.e., $a_{k}, b_{k}, \ldots, n_{k}$ ) are real. The two-dimensional version of the configuration is shown in Fig. 2(b). This time $f_{\text {out }}(u, v)$ is related to $f_{\text {in }}\left(u^{\prime}, v^{\prime}\right)$ through a linear transformation [as in Eq. (5)] whose kernel is expressed as

$$
\begin{aligned}
T\left(u, v, u^{\prime} v^{\prime}\right)= & \iint \cdots \iint \mathrm{d} u_{1} \mathrm{~d} v_{1} \cdots \mathrm{d} u_{M-1} \mathrm{~d} v_{M-1} \\
& \times h_{1}\left(u^{\prime}, v^{\prime}\right) h_{2}\left(u_{1}, v_{1}\right) \cdots h_{M+1}(u, v) \\
& \times Q_{M}\left(u, v, u_{M-1}\right. \\
& \left.v_{M-1}\right) \cdots Q_{1}\left(u_{1}, v_{1}, u^{\prime}, v^{\prime}\right)
\end{aligned}
$$


[As in the one-dimensional case, we also scale the variables of all $Q_{l}\left(x, y, x^{\prime}, y^{\prime}\right), l=1, \ldots, M$, with $s$ and obtain the dimensionless variables $u, v, u^{\prime}$, and $v^{\prime}$.] The error is now defined as

$$
\begin{aligned}
e= & \iiint \int \mid T_{d}\left(u, v, u^{\prime}, v^{\prime}\right) \\
& -\left.T\left(u, v, u^{\prime}, v^{\prime}\right)\right|^{2} \mathrm{~d} u \mathrm{~d} v \mathrm{~d} u^{\prime} \mathrm{d} v^{\prime} .
\end{aligned}
$$

Again our purpose is to choose the filters and the parameters of the two-dimensional quadratic-phase system kernels in order to minimize this error, provided that $T\left(u, v, u^{\prime}, v^{\prime}\right)$ is in the form given in Eq. (9).

\section{B. Reduction of the Problem to Its Canonical Form}

Let us first consider the one-dimensional problem. It is possible to show that the configuration in Fig. 2(a) can be converted to that in Fig. 3(a) by appropriately choosing the hatted filter functions $\hat{h}_{1}(u), \hat{h}_{2}(u), \ldots, \hat{h}_{M+1}(u)$ and the real constant $c$. Thus we see that it is possible to cast the quadratic-phase-system-based repeated-filtering problems into an equivalent but simpler form involving only ordinary Fourier transforms. A weaker form of this result was stated in Refs. 38 and 39.

Let us now look at the two-dimensional version of the problem. This time it is possible to show that the configuration in Fig. 2(b) can be converted to that in Fig. 3(b) by appropriately choosing the hatted filter functions $\hat{h}_{1}(u, v), \hat{h}_{2}(u, v), \ldots, \hat{h}_{M+1}(u, v)$ and the real constants $p, r, s$, and $t$. Thus again the quadratic-phase-systembased repeated-filtering problem is reduced to repeated filtering in consecutive ordinary Fourier stages.

The demonstration of these results is straightforward but involves an amount of algebra. Starting with the associated kernel expressions in Eqs. (6) and (9), we see that, by changing the integration variables properly and absorbing the quadratic exponential terms in the filter functions, we can convert these kernel expressions into the ones whose block diagrams are shown in Fig. 3.

Since fractional Fourier transformations are a special case of quadratic-phase systems, repeated filtering in consecutive fractional Fourier domains can also be reduced to repeated filtering in consecutive conventional Fourier domains (in both one-dimensional and two-dimensional sys-

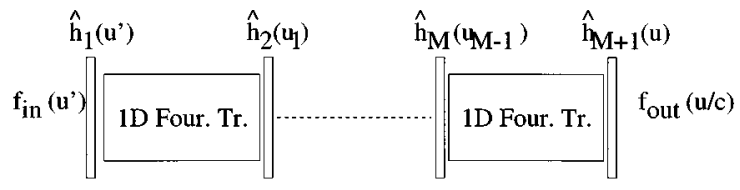

(a)

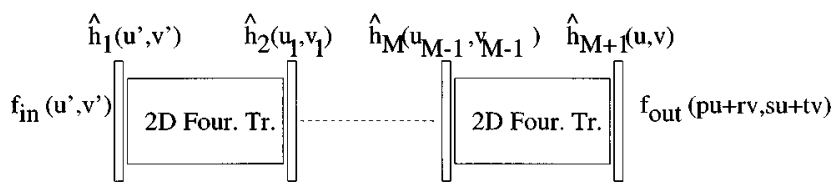

(b)

Fig. 3. Canonical forms for (a) the 1D repeated-filtering configuration in Fig. 2(a), (b) the 2D repeated-filtering configuration in Fig. 2(b). tems). This does not, however, reduce the conceptual and practical importance of the fractional Fourier transformation (or of quadratic-phase systems). First of all, optical and digital implementations of the fractional Fourier transformation (or quadratic-phase systems) are not more difficult than those of the ordinary Fourier transformation. Thus filtering in different domains does not imply extra work. Second, the implementation of necessary filters may be easier at a specific domain. For example, in chirp elimination ${ }^{1}$ the filters necessary in fractional domains are simply the mask filters. However, in the space and spatial frequency domains the filters would have to be complex functions. Furthermore, the accuracy needed to implement a filter in one domain may be less than that needed in others. Thus repeated filtering in consecutive fractional Fourier domains may offer better noise redundancy and may be more robust. In conclusion, the equivalence of repeated filtering in any consecutive quadratic-phase system domains should be used to increase the number of possible candidates for a specific physical realization. From now on we will concentrate on the configurations in Fig. 3 and also ignore the scale factor $c$ at the output in Fig. 3(a), or the parameters $p, r$, $s$, and $t$ at the output in Fig. 3(b), since these can be easily handled. The filter profiles in Figs. 2(a) and 2(b) can be easily recovered in terms of the ones in Figs. 3(a) and $3(\mathrm{~b})$, respectively.

\section{Discretization of the Problem}

Let us first look at the one-dimensional repeated-filtering configuration in Fig. 3(a). We assume that the maximum value for the space-bandwidth products of the signals is $N$. Then we sample $f_{\text {in }}(u), f_{\text {out }}(u), \hat{h}_{1}(u), \hat{h}_{2}(u), \ldots$, $\hat{h}_{M+1}(u)$ to obtain the vectors $\bar{f}_{\text {in }}, \bar{f}_{\text {out }}, \bar{h}_{1}, \bar{h}_{2}, \ldots, \bar{h}_{M+1}$, each of which has $N$ elements, and the matrices $\hat{T}$ and $\hat{T}_{d}$, which have $N \times N$ elements. We can now relate $\bar{f}_{\text {out }}$ to $\bar{f}_{\text {in }}$ through

$$
\begin{aligned}
\bar{f}_{\text {out }} & =\hat{T} \bar{f}_{\text {in }}, \\
\hat{T} & =\hat{\Lambda}_{M+1} \hat{F} \hat{\Lambda}_{M} \hat{F} \cdots \hat{F} \hat{\Lambda}_{2} \hat{F} \hat{\Lambda}_{1},
\end{aligned}
$$

where $\hat{\Lambda}_{k}$ is an $N \times N$ diagonal matrix with its diagonal elements equal to the components of $\bar{h}_{k}$ and $\hat{F}$ is an $N$ $\times N$ discrete Fourier transformation matrix. Thus, for one-dimensional systems, we can state our repeatedfiltering problem so as to choose the vectors $\bar{h}_{1}, \bar{h}_{2}, \ldots$, $\bar{h}_{M+1}$ to minimize the error function

$$
e=\sum_{k=1}^{N} \sum_{l=1}^{N}\left|\left(\hat{T}_{d}\right)_{k l}-\hat{T}_{k l}\right|^{2} .
$$

This error function is the discrete version of the error function expressed in Eq. (7). The matrix $\hat{T}_{d}$ corresponds to the kernel of the linear transformation in Eq. (4).

Let us now consider the two-dimensional case in Fig. 3(b). Here we again assume that the maximum value for the space-bandwidth products of the signals is $N$. Then we sample $f_{\text {in }}\left(u, u^{\prime}\right), f_{\text {out }}\left(u, u^{\prime}\right), \hat{h}_{1}\left(u, u^{\prime}\right)$, $\hat{h}_{2}\left(u, u^{\prime}\right), \ldots, \hat{h}_{M+1}\left(u, u^{\prime}\right)$ to obtain the corresponding $N \times N$ square matrices $\hat{f}_{\text {in }}, \hat{f}_{\text {out }}, \hat{h}_{1}, \hat{h}_{2}, \ldots, \hat{h}_{M+1}$ and 
the four-dimensional tensors $\widetilde{T}$ and $\widetilde{T}_{d}$. Similarly, we now relate $\hat{f}_{\text {out }}$ to $\hat{f}_{\text {in }}$ through

$$
\begin{aligned}
\hat{f}_{\text {out }} & =\widetilde{T} \hat{f}_{\text {in }}, \\
\widetilde{T} & =\widetilde{\Lambda}_{M+1} \widetilde{F}_{2 \mathrm{D}} \widetilde{\Lambda}_{M} \widetilde{F}_{2 \mathrm{D}} \cdots \widetilde{F}_{2 \mathrm{D}} \widetilde{\Lambda}_{2} \widetilde{F}_{2 \mathrm{D}} \widetilde{\Lambda}_{1},
\end{aligned}
$$

where the four-dimensional tensor $\widetilde{T}$ represents the kernel of the linear transformation between the twodimensional input and output signals, $\widetilde{F}_{2 \mathrm{D}}$ represents the conventional two-dimensional discrete Fourier transformation, and $\tilde{\Lambda}_{k}$ is related to the $k$ th filter through

$$
\left(\widetilde{\Lambda}_{k}\right)_{l m j k}=\left\{\begin{array}{ll}
\left(\hat{h}_{k}\right)_{l m} & \text { if } l=j \text { and } m=k \\
0 & \text { otherwise }
\end{array} .\right.
$$

We define the multiplication of a four-dimensional tensor with a two-dimensional matrix in Eqs. (13) as

$$
(\tilde{K} \hat{f})_{u v}=\sum_{l} \sum_{m} \tilde{K}_{u v l m} \hat{f}_{l m}
$$

and the multiplication of two four-dimensional tensors as

$$
(\tilde{K} \widetilde{L})_{u v j k}=\sum_{l} \sum_{m} \widetilde{K}_{u v l m} \widetilde{L}_{l m j k} .
$$

Our problem is once again to choose the matrices $\hat{h}_{1}$, $\hat{h}_{2}, \ldots, \hat{h}_{M+1}$ to minimize the error function

$$
e=\sum_{u=1}^{N} \sum_{v=1}^{N} \sum_{j=1}^{N} \sum_{k=1}^{N}\left|\left(\widetilde{T}_{d}\right)_{u v j k}-\widetilde{T}_{u v j k}\right|^{2} .
$$

This time the error function corresponds to the discrete version of the error function expressed in Eq. (10). The four-dimensional tensor $\widetilde{T}_{d}$ corresponds to the kernel of the linear transformation in Eq. (5).

\section{Solution of the Problem}

Let us again first consider the one-dimensional case. We see from Eq. (11b) that $\hat{T}$ depends on the filters in a highly nonlinear manner. Thus we cannot obtain the closed forms of the filter functions that minimize the error expression in Eq. (12). For this reason we obtain the filters by an iterative algorithm. We first initialize all the filters to some convenient values. Then, starting with the first filter, we assume that all the filter profiles apart from $\bar{h}_{k}$ are known, and we calculate the optimum value for the $k$ th filter in terms of the remaining filters. We then pass on to the $(k+1)$ th filter. When we reach $k=M+1$ and obtain the optimum profile for $\bar{h}_{M+1}$, we set $k=1$ and start again with the first filter. We continue this until the iteration converges.

An important step of the algorithm is to calculate the $k$ th filter in terms of the other filters so as to bring $\hat{T}$ as close to $\hat{T}_{d}$ as possible. Defining $\hat{A}$ and $\hat{B}$ as

$$
\begin{aligned}
& \hat{A}=\hat{\Lambda}_{M+1} \hat{F} \cdots \hat{F} \hat{\Lambda}_{k+1} \hat{F}, \\
& \hat{B}=\hat{F} \hat{\Lambda}_{k-1} \hat{F} \cdots \hat{F} \hat{\Lambda}_{1},
\end{aligned}
$$

we can rewrite $\hat{T}$ in Eq. (11b) in the form

$$
\hat{T}=\hat{A} \hat{\Lambda}_{k} \hat{B} \text {. }
$$

We now show how to find the filter $\bar{h}_{k}$ (whose elements are simply the diagonal elements of $\hat{\Lambda}_{k}$ ) that minimizes the error $e$ in Eq. (12). We express the $m$ th component of the $k$ th filter as

$$
h_{k m}=h_{k m}^{r}+j h_{k m}^{i} .
$$

Then we differentiate the error with respect to the real and imaginary parts of these components and equate the resulting expressions to zero:

$$
\frac{\delta e}{\delta h_{k m}^{r}}=0, \quad \frac{\delta e}{\delta h_{k m}^{i}}=0, \quad m=1,2, \ldots, N .
$$

If we use the definition of $e$ in Eq. (12), then, after some algebra, these two conditions imply that

$$
\hat{D} \bar{h}_{k}=\bar{c} .
$$

In this equation

$$
\hat{D}=\left(\hat{A}^{\mathrm{H}} \hat{A}\right) \otimes\left(\hat{B} \hat{B}^{\mathrm{H}}\right)^{\mathrm{T}},
$$

where the operator $\otimes$ corresponds to elementwise multiplication of two matrices, and we get the elements of $\bar{c}$ through

$$
\bar{c}_{l}=\left(\hat{A}^{\mathrm{H}} \hat{T}_{d} \hat{B}^{\mathrm{H}}\right)_{l l} .
$$

[In Eqs. (24) and (25), $\hat{A}^{\mathrm{H}}$ and $\hat{A}^{\mathrm{T}}$ correspond to the Hermitian transpose and the ordinary transpose of $\hat{A}$, respectively.] Thus, as evident from Eq. (23), we have $N$ linear equations with $N$ unknowns, from which we can solve for the filter coefficients. As a result, given all the filter profiles apart from $\bar{h}_{k}$, we know how to obtain the filter $\bar{h}_{k}$. Once this subroutine is established, the iteration proceeds as described in the first paragraph of this subsection.

The corresponding subroutine for the two-dimensional case is established similarly. Now we define $\widetilde{A}$ and $\widetilde{B}$ as

$$
\begin{aligned}
& \widetilde{A}=\widetilde{\Lambda}_{M+1} \widetilde{F}_{2 \mathrm{D}} \cdots \widetilde{F}_{2 \mathrm{D}} \widetilde{\Lambda}_{k+1} \widetilde{F}_{2 \mathrm{D}}, \\
& \widetilde{B}=\widetilde{F}_{2 \mathrm{D}} \widetilde{\Lambda}_{k-1} \widetilde{F}_{2 \mathrm{D}} \cdots \widetilde{F}_{2 \mathrm{D}} \widetilde{\Lambda}_{1}
\end{aligned}
$$

and rewrite $\widetilde{T}$ in Eq. (13b) as

$$
\widetilde{T}=\widetilde{A} \widetilde{\Lambda}_{k} \widetilde{B} .
$$

After some algebra we then obtain

$$
\widetilde{D} \hat{h}_{k}=\hat{c} \text {. }
$$

In this equation

$$
\widetilde{D}=\left(\widetilde{A}^{\mathrm{H}} \widetilde{A}\right) \otimes\left(\widetilde{B} \widetilde{B}^{\mathrm{H}}\right)^{\mathrm{T}},
$$

and we get the elements of $\hat{c}$ through

$$
\hat{c}_{l m}=\left(\widetilde{A}^{\mathrm{H}} \widetilde{T}_{d} \widetilde{B}^{\mathrm{H}}\right)_{l m l m} .
$$

$\left[\widetilde{A}^{\mathrm{H}}\right.$ is the Hermitian transpose of the four-dimensional tensor $\tilde{A}$. That is, the $(u, v, l, m)$ th element of $\widetilde{A}^{\mathrm{H}}$ is equal to the conjugate of the $(l, m, u, v)$ th element of $\tilde{A}$. Similarly, the $(u, v, l, m)$ th element of $\widetilde{A}^{\mathrm{T}}$ is equal to the $(l, m, u, v)$ th element of $\widetilde{A}$.] Thus, as evident from Eq. (29), we have $N^{2}$ linear equations with $N^{2}$ unknowns, from which we can solve for the filter coefficients. As a result, given all the filter profiles apart from $\hat{h}_{k}$, we know 
how to obtain the filter $\hat{h}_{k}$, and the iteration proceeds as described in the first paragraph of this subsection.

The iterative algorithm that we propose always converges to a minimum point. However, because of the nonlinear nature of the problem, the algorithm may not, and in general will not, converge to the global minimum but rather will converge to a local minimum. In practice, we ran the algorithm for several different initial starting points and chose the run resulting in the smallest MSE. We did not overly concern ourselves with determining the global minimum, since the values that we obtained already represented satisfactory performance figures. Thus our results represent achievable, but not necessarily the best possible, solutions. Better solutions may be obtained by using more sophisticated optimization algorithms.

\section{E. Kernel Synthesis with Repeated Phase-Only Filtering}

As phase-only filters are sometimes preferred to those with arbitrary complex amplitudes, we will also consider the repeated-filtering problem under this constraint. More specifically, we will restrict the filters in Fig. 3 to be phase-only filters.

For the solution of the repeated phase-only filtering problem, the discussion of Subsection 3.D applies identically until Eq. (23). Now, since the filter coefficients are restricted to being phase only, $\hat{D}$ in that equation reduces to the identity matrix, so that

$$
\left(\bar{h}_{k}\right)_{l}=\left(\hat{A}^{\mathrm{H}} \hat{T}_{d} \hat{B}^{\mathrm{H}}\right)_{l l} .
$$

Once we obtain $\bar{h}_{k}$ in terms of the other filters by using this equation, we then force it to be a phase-only filter by dividing each of its element by its magnitude, i.e.,

$$
\frac{\left(\bar{h}_{k}\right)_{l}}{\left|\left(\bar{h}_{k}\right)_{l}\right|} \rightarrow\left(\bar{h}_{k}\right)_{l}, \quad l, m=1,2, \ldots, N .
$$

The iteration then proceeds as in the first paragraph of Subsection 3.D. Similarly, in the two-dimensional case $\hat{h}_{k}$ is obtained as

$$
\left(\hat{h}_{k}\right)_{l m}=\left(\widetilde{A}^{\mathrm{H}} \widetilde{T}_{d} \widetilde{B}^{\mathrm{H}}\right)_{l m l m}
$$

and is forced to be a phase-only filter according to

$$
\frac{\left(\hat{h}_{k}\right)_{l m}}{\left|\left(\hat{h}_{k}\right)_{l m}\right|} \rightarrow\left(\hat{h}_{k}\right)_{l m}, \quad l, m=1,2, \ldots, N .
$$

Note that in the phase-only case there are no equations to be solved, unlike the general case, where $N$ equations in $N$ unknowns were solved for the one-dimensional case and $N^{2}$ equations in $N^{2}$ unknowns were solved for the two-dimensional case. Thus fewer computations are needed in the phase-only case.

The phase-only case is, of course, less flexible than the general case but may nevertheless be found satisfactory under a variety of circumstances.

\section{APPLICATIONS}

We see from Eqs. (4) and (5) that linear systems are completely characterized by their transformation kernels.
The matrix $\hat{T}_{d}$ or the four-dimensional tensor $\widetilde{T}_{d}$ can be considered as the sampled versions of the kernels $T_{d}\left(u, u^{\prime}\right)$ and $T_{d}\left(u, v, u^{\prime}, v^{\prime}\right)$, so that the products $\bar{f}_{\text {out }}$ $=\hat{T}_{d} \bar{f}_{\text {in }}$ and $\hat{f}_{\text {out }}=\widetilde{T}_{d} \hat{f}_{\text {in }}$ represent a discrete approximation of the continuous linear systems given in Eqs. (4) and (5). Alternatively, these products may represent a priori discrete linear systems or simply matrix-vector or tensor-matrix products that we wish to evaluate. Thus we will discuss the applications of our repeated-filtering method from two broad perspectives. One of them is about the implementations of linear systems, and the other is about matrix algebra operations.

\section{A. Implementations of General Linear Systems}

In some applications we may want to implement a desired linear system in order to observe a certain effect on the input. However, the optical and digital implementations of general linear systems are costly. If we can obtain satisfactory approximations to a given linear system by using a moderate number of filters in our repeated-filtering configuration, we can considerably reduce the implementation costs. We will consider two examples in which this is possible.

\section{Signal Restoration}

Sometimes we may want to restore a desired signal that is degraded by a known system and/or by a noise term. With this aim in mind, we search for an appropriate operator that minimizes the effect of degradation and noise. This operator strongly depends on the observation model, the design criteria used, and the prior knowledge available about the desired signal and noise. We assume a signal observation model of the form

$$
\bar{y}=\hat{H} \bar{x}+\bar{n} .
$$

In this equation $\bar{y}, \bar{x}$, and $\bar{n}$ are the column vectors representing the output, input, and noise processes, respectively, and $\hat{H}$ is the matrix characterizing the degradation process. We further assume that the correlation matrices of the signal and the noise are known and that the noise is independent of the signal $\bar{x}$ and has zero mean. Our aim is to minimize the MSE, defined as

$$
\sigma^{2}=\frac{1}{N} E\left[\left(\bar{x}-\bar{x}_{e}\right)^{\mathrm{H}}\left(\bar{x}-\bar{x}_{e}\right)\right] .
$$

There are now two basic approaches at our disposal.

1. We may restrict the estimate to be of the form

$$
\bar{x}_{e}=\left[\hat{\Lambda}_{M+1} \hat{F} \hat{\Lambda}_{M} \hat{F} \cdots \hat{F} \hat{\Lambda}_{2} \hat{F} \hat{\Lambda}_{1}\right] \bar{y}
$$

and seek the diagonal matrices $\hat{\Lambda}_{M+1}, \hat{\Lambda}_{M}, \ldots, \hat{\Lambda}_{2}, \hat{\Lambda}_{1}$ (or the filters $\bar{h}_{M+1}, \bar{h}_{M}, \ldots, \bar{h}_{2}, \bar{h}_{1}$ ) that minimize the MSE. This approach was proposed in Ref. 10 and was applied to three important degradation models. These were the effect of multiple random-phase plates (which may model surface imperfections in optical systems), atmospheric turbulence, and nonconstant-velocity moving-camera blur. In all of the examples in Ref. 10, it was seen that when the proposed approach was compared with singledomain filtering methods, significant improvements in performance were obtained with only modest increases in processing time. The method of that paper was also com- 
pared with the optimum linear estimation method, and it was seen that its use might result in significant computational savings while still yielding acceptable performance.

2. Alternatively, we can first obtain the optimum linear estimation operator that gives the smallest MSE among all linear operators:

$$
\bar{x}_{e}=\hat{G}_{\mathrm{opt}} \bar{y} \text {. }
$$

As discussed above, direct implementation of this operator is costly. Here we will consider approximating $\hat{G}_{\mathrm{opt}}$ with our repeated-filtering configuration. To illustrate this concept, we will approximately synthesize the optimum linear estimation kernel that corresponds to spacevarying atmospheric turbulence for purposes of illustration. The system degradation that corresponds to spacevarying atmospheric turbulence is the result of inhomogeneous statistical properties of the turbulent media. ${ }^{40}$ The one-dimensional kernel is given by

$$
h\left(x ; x^{\prime}\right)=\exp \left[-\pi \alpha^{2}(x)\left(x-x^{\prime}\right)^{2}\right],
$$

where $\alpha(x)$ is a function of $x$ characterizing the distribution of the physical parameters in the turbulent atmosphere. It is convenient to employ piecewise-constant approximation. Here we consider seven intervals numbered by $k=1,2, \ldots, 7$ such that $\alpha(x)=\alpha_{0}+\beta_{k}$, where $\alpha_{0}=0.1$ and $\beta_{k} \ll \alpha_{0}$. We obtain the corresponding optimum linear estimation matrix $\hat{G}_{\text {opt }}$ and illustrate the performance of our repeated-filtering configuration by using the normalized error $e_{n}$, defined as

$$
e_{n}=e /\left\|\hat{T}_{d}\right\|^{2},
$$

where $e$ is defined in Eq. (12) and $\hat{T}_{d}=\hat{G}_{\text {opt }}$. With $N$ $=128$ and initializing all the diagonal matrices in Eq. (11b) to the identity matrix, we obtain the normalizederror values through the iterative algorithm proposed in Section 3. The $e_{n}$ values for the sinusoidal-type input signal (i.e., the desired signal is a sinusoidal whose frequency, phase, and amplitude are random) corrupted with no additive noise are plotted in Fig. 4 for different numbers of filters.

It is important to emphasize that the method that we propose is general and not dependent on any of the specific assumptions made in this problem. Typically, linear

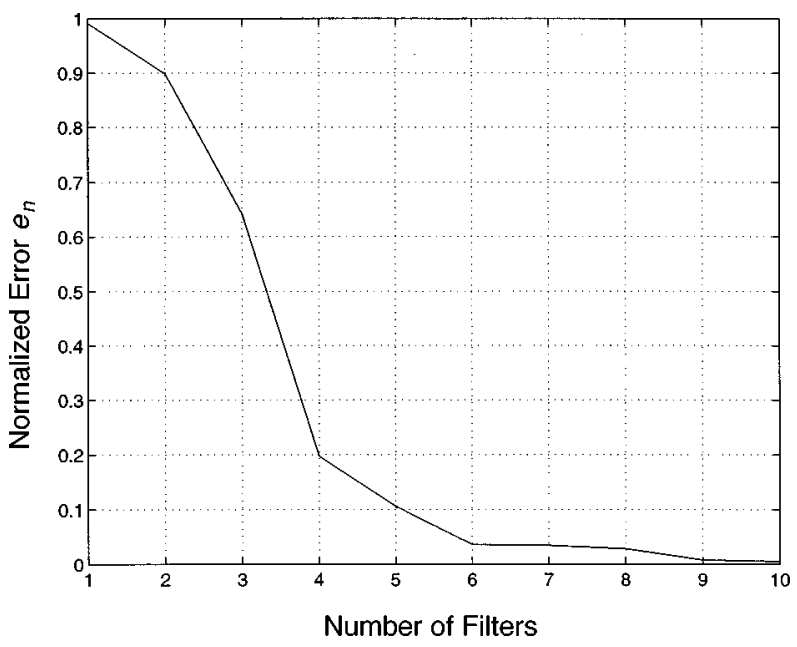

Fig. 4. Normalized error $e_{n}$ versus number of filters in the repeated-filtering configuration for the signal restoration example. restoration, recovery, reconstruction, and many other signal and image processing problems can be thought to consist of two distinct processes. The first of these is to obtain the optimal linear restoration, reconstruction, enhancement, etc., operator. The second is to implement this operator. We deal with this second part of the problem regardless of how and under which assumptions the optimal linear operator to be implemented is obtained. Thus the above example is only one of a large class of applications that may benefit from the method.

\section{Moment Generation}

This time we will apply the repeated-filtering configuration for generating the moments of signals. The moments of a signal are important in several signal processing applications such as feature extraction. ${ }^{40,41}$

Let $f_{\text {in }}(x) \geqslant 0$ be a real bounded function that is zero outside a finite interval. Without loss of generality it is assumed in Ref. 40 that $f_{\text {in }}(x)$ is nonzero only in the interval $-1<x<1$. Then the $i$ th-order moment of $f_{\text {in }}(x)$ is defined as

$$
M_{i}=\int_{-1}^{1} f_{\text {in }}(x) x^{i} \mathrm{~d} x .
$$

The discrete form of this equation is

$$
\bar{M}=\hat{T}_{d} \bar{f}_{\text {in }},
$$

where $\bar{f}_{\text {in }}$ is the column vector corresponding to the sampled version of $f_{\text {in }}(x)$, the $i$ th row of the matrix $\hat{T}_{d}$ corresponds to the sampled version of the function $x^{i-1}$, and the $i$ th element of the vector $\bar{M}$ is equal to the $(i-1)$ th moment of the input signal (i.e., the first element of $\bar{M}$ is equal to the zeroth moment, the second element of $\bar{M}$ is equal to the first moment, and so on).

Thus all of the moments of $\bar{f}_{\text {in }}$ can be calculated simultaneously by multiplying it with the matrix $\hat{T}_{d}$. We will synthesize this matrix with the repeated-filtering method. We again show our results in terms of the normalized error $e_{n}$ defined in Eq. (41). With $N=128$ [and starting the iterative algorithm proposed in Subsection 3.D with all the diagonal matrices in Eq. (11b) initialized to the identity matrix], we obtain the plot in Fig. 5. In this plot, for example with only two filters, $e_{n}=0.737$. This value reduces to 0.038 with four filters, and it further reduces to 0.009 with five filters.

Reduction of the MSE may not always translate into a better output. For this reason, in Fig. 6 we present an illustrative example that helps us to visualize the performance of our repeated-filtering method (which minimizes the MSE) more clearly. In Fig. 6(b) we show the moment space representation (i.e., all 128 moments, starting with the zeroth moment going up to the 127th moment) of the sine function shown in Fig. 6(a). Then, for this specific moment generation problem, we calculate the optimum (in terms of MSE criteria) single filter and the repeatedfiltering configuration with $M=5$ filters and show their approximation for the moment space representation of the sine function in Figs. 6(c) and 6(d), respectively. We see from these plots that use of $M=5$ filters is clearly superior to use of only one filter, and in some situations the 


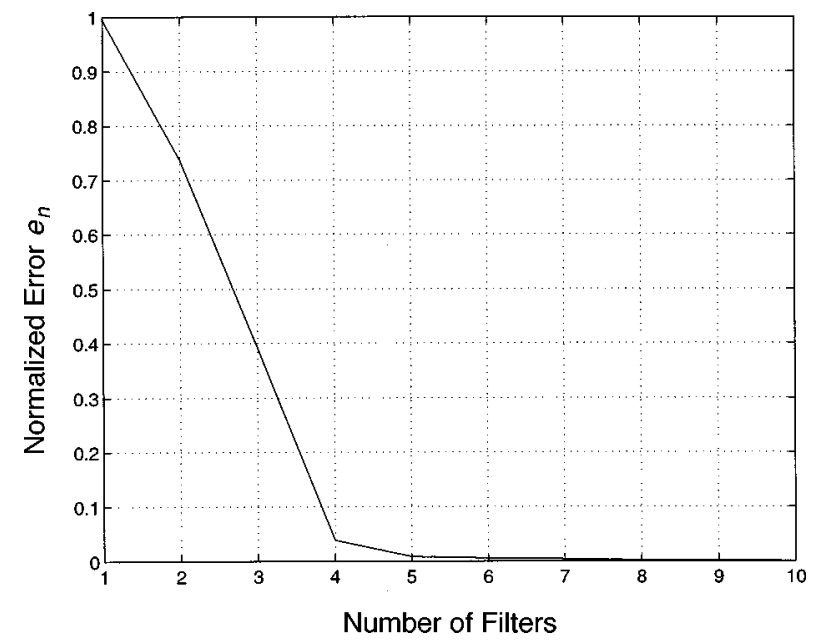

Fig. 5. Same as Fig. 4, but for the moment generation example.

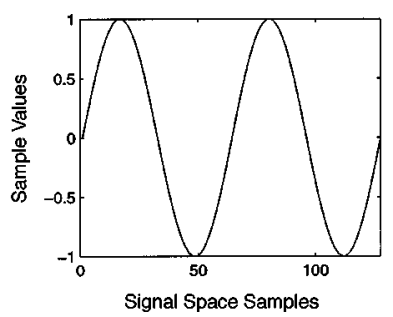

(a)

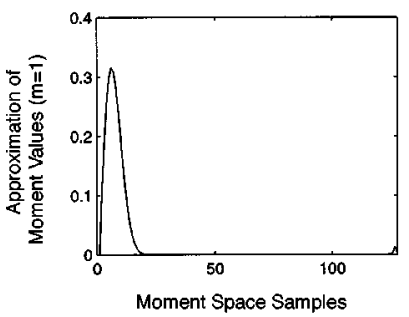

(c)

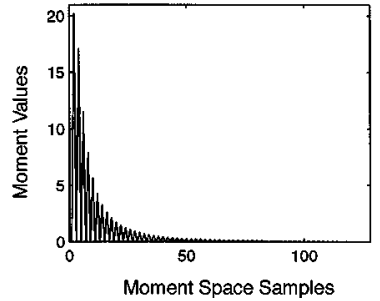

(b)

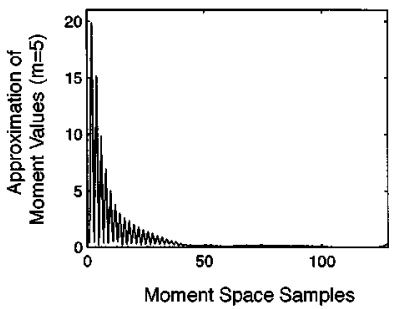

(d)
Fig. 6. (a) Sinusoidal function, (b) moment space representation of the sine function in (a), (c) approximation of the moment space representation in (b) obtained with one filter $(M=1)$, (d) approximation of the moment space representation in (b) obtained with five filters $(M=5)$.

configuration with $M=5$ filters can be considered as sufficient for approximating the desired linear system that generates moment space representations of signals.

We can similarly generate the moments of an image. Let $f_{\text {in }}(x, y) \geqslant 0$ be a real bounded function that is zero outside a finite region. We assume this time that $f_{\text {in }}(x, y)$ is nonzero only in the region defined by $-1<x$ $<1$ and $-1<y<1$. Then the $(i, l)$ th-order moment of $f_{\text {in }}(x, y)$ is defined as

$$
M_{i, l}=\int_{-1}^{1} \int_{-1}^{1} f_{\mathrm{in}}(x, y) x^{i} y^{l} \mathrm{~d} x \mathrm{~d} y .
$$

Similar to that of the one-dimensional case, the discrete form of this equation is

$$
\hat{M}=\widetilde{T}_{d} \hat{f}_{\text {in }},
$$

where $\hat{f}_{\text {in }}$ is the matrix corresponding to the sampled version of $f_{\text {in }}(x, y)$, the $(i, l)$ th matrix of the four- dimensional tensor $\widetilde{T}_{d}$ corresponds to the sampled version of the function $x^{i-1} y^{l-1}$, and the $(i, l)$ th element of the vector $\bar{M}$ is equal to the $(i-1, l-1)$ th moment of $\hat{f}_{\text {in }}$. The desired four-dimensional tensor is separable. That is, the elements of $\widetilde{T}_{d}$ can be written as the multiplication of the elements of the two matrices $\hat{T}_{d x}$ and $\hat{T}_{d y}$. These matrices are of the same form as that of the matrix appearing in Eq. (43). Thus, once the optimal filter profiles are obtained for the one-dimensional case, those for the two-dimensional case can be immediately synthesized.

Various optical setups for calculating the moments of an image have been suggested. However, these typically calculate only one moment at a time. ${ }^{41}$ In other words, to compute, for example, 20 moments of an image, each time we have to modify the setup (i.e., we have to change the spatial filter in the setup accordingly) and repeat the experiment 20 times (or we have to employ 20 different optical setups operating in parallel, or we have to divide the aperture into 20 different channels). However, with our method we can simultaneously calculate the $128 \times 128$ moments without modifying the setup. Thus the full moment space representation of images is obtained at once. The system consists of a moderate number of stages and requires an optical space-bandwidth product equal to the number of pixels in the image $(128 \times 128$ in the above example). This is in contrast to matrix-vector-multiplier or multifacet-type architectures, which both require optical space-bandwidth products equal to the square of the number of pixels.

\section{B. Matrix Algebra Operations}

In Subsection $4 . \mathrm{A}$ we assumed that $\bar{f}_{\text {out }}=\hat{T}_{d} \bar{f}_{\text {in }}$ or $\hat{f}_{\text {out }}$ $=\widetilde{T}_{d} \hat{f}_{\text {in }}$ represent the discrete approximations of continuous linear systems. We will now interpret these as representing an a priori discrete linear system or simply a matrix-vector product that we wish to compute. We will again try to obtain satisfactory approximations to the desired matrices or tensors by using a moderate number of filters in our repeated-filtering configuration. Thus we will be able to realize these matrix-vector products more efficiently.

\section{Synthesis of the Hadamard Transformation}

The Hadamard transformation is one of the standard unitary transformations in signal processing. Its definition and properties may be found in Ref. 40. Here we synthesize this transformation with our repeated-filtering method, and for $N=128$ we find the normalized error $e_{n}$ in Eq. (41) to be 0.01 with five filters. [We obtain this value through the iterative algorithm when the diagonal matrices in Eq. (11b) are initialized to the identity matrix.]

When we restrict ourselves to phase-only filters, we obtain $e_{n}=0.214$ with seven filters and $e_{n}=0.028$ with 11 filters. We further decrease $e_{n}$ to 0.005 with 15 filters. This shows that if we do not restrict the filter type, the same error can be achieved with a smaller number of filters. The cost of the repeated-filtering configuration 
depends on both the filter type and the number of filters used. Given the relevant cost functions, one can determine whether a smaller number of arbitrary filters or a relatively larger number of phase-only filters results in overall less cost.

It may have been possible to come up with an optical setup with a comparable or even a fewer number of stages through ingenuity and invention. Our approach provides, on the other hand, a systematic way of obtaining such an implementation. This would be of great utility, especially in those cases where the structure of the transformation matrix is not simple, or even when we are confronted with a matrix supplied in numerical form for which no easily discernible structure is apparent.

\section{Synthesis of Optical Interconnection Architectures}

Here we will consider the problem of realizing one-to-one interconnection patterns between $N$ input and $N$ output channels ${ }^{13,42}$ and will try to implement these interconnection patterns with repeated phase-only filtering. There are many different ways of achieving this aim. Some of these have been compared in Refs. 13 and 42, and the conclusion is reached that the multistage architectures based on regular patterns such as the perfect shuffle or the Banyan are most favorable. The repeated-filtering-based approach that we will discuss is essentially analogous to multistage architectures. However, not only does our method provide a systematic way of designing such systems, but the implementation of such systems may be more convenient and/or cheaper, since the present approach is based on the use of conventional spatial filters rather than micro-optical elements.

Any one-to-one interconnection architecture between $N$ input and $N$ output channels is characterized by its associated $N \times N$ permutation matrix. In such a matrix every row and column has only one nonzero element, which is equal to unity. We synthesize the interconnection architectures by synthesizing their associated permutation matrices.

First, we consider the reverse perfect shuffle architecture shown in Fig. 7. We find that this interconnection pattern can be synthesized exactly $\left(e_{n}=0\right)$ by using six phase-only filters in five consecutive domains. [The coefficients of the filters in Eq. (11b) were initialized to $\exp (j \pi / 25)$.] We have also considered a large number of interconnection patterns that do not exhibit any obvious regularity. In all cases these patterns could be realized

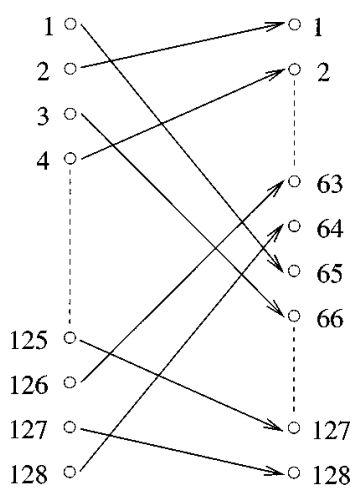

Fig. 7. Reverse perfect shuffle interconnection architecture.

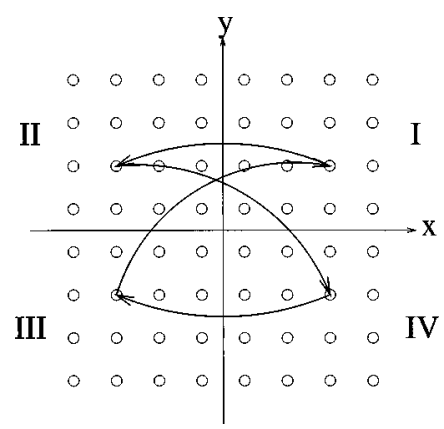

Fig. 8. 64 points are mapped to 64 points. The points lying in the first quadrant are mapped to the points in the third quadrant, which are symmetric to them with respect to the origin. The points lying in the fourth quadrant are similarly mapped to points in the second quadrant. However, the points lying in the second quadrant are mapped to points in the first quadrant, which are symmetric to them with respect to the $y$ axis. The points in the third quadrant are likewise mapped to the fourth quadrant.

with a moderate number of filters. (We do not explicitly include specific examples of these, to avoid taking up space by specifying the interconnection pattern.) Conventional multistage permutation network architectures can realize arbitrary permutations in $O(\log N)$ stages. Extensive numerical experimentation on many different arbitrary permutation matrices indicates that the proposed method is also able to realize these in a similar number of stages. Although it is not difficult to achieve $\hat{T}=\hat{T}_{d}$ with a moderate number of stages, in most cases it is possible to get away with an even smaller number of stages, since, as a result of the digital nature of such systems, a considerable deviation of $\hat{T}$ from $\hat{T}_{d}$ can be tolerated while still retaining an acceptable eye pattern.

Let us also consider the two-dimensional case. In the event that the four-dimensional tensor mapping the input to the output is separable, the elements of $\widetilde{T}_{d}$ can be written as the multiplication of the elements of the two matrices $\hat{T}_{d x}$ and $\hat{T}_{d y}$ [i.e., $\left(\widetilde{T}_{d}\right)_{u v l m}=\left(\hat{T}_{d x}\right)_{u l}\left(\hat{T}_{d y}\right)_{v m}$ for all $u, v, l$, and $m]$. In this case the matrices $\hat{T}_{d x}$ and $\hat{T}_{d y}$ correspond to the $N \times N$ matrices appearing in the onedimensional case, and the two-dimensional mapping can be realized in a relatively straightforward manner. Instead of the separable case, we have considered the direct implementation of the more general nonseparable case. For the sake of illustration, we assume a nonseparable mapping, shown in Fig. 8. This time $e$ in Eq. (41) corresponds to the one defined in Eq. (17), and $\hat{T}_{d}$ stands for the four-dimensional kernel of the interconnection architecture in Fig. 8. In this example seven phase-only filters were sufficient to obtain an exact representation $\left(e_{n}=0\right)$. [The coefficients of the filters in Eq. (13b) were initialized to unity.] If one is willing to tolerate greater errors, the number of filters needed may be reduced.

\section{DISCUSSION}

In Section 4 we interpreted the discrete equations $\bar{f}_{\text {out }}$ $=\hat{T}_{d} \bar{f}_{\text {in }}$ and $\hat{f}_{\text {out }}=\widetilde{T}_{d} \hat{f}_{\text {in }}$ in two different ways. However, these interpretations are not different from each other. Any matrix or tensor in matrix algebra can be considered as representing the kernel of a linear system, 
and any discrete form of the kernel of a linear system can be interpreted as a specific matrix or tensor in matrix algebra.

In this paper what we essentially did was to synthesize a given matrix or a four-dimensional tensor with our repeated-filtering method. The synthesized matrices or tensors may find applications in both linear system implementations and matrix algebra operations. We have tried to obtain satisfactory approximations to the desired matrices or tensors by using a moderate number of filters in our repeated-filtering configuration. Thus we were able to implement more efficiently either the linear systems corresponding to these matrices or tensors, or the matrix-vector multiplications. The examples that we presented should be considered merely as illustrations; doubtless the method can be applied to a wide range of situations.

While implementing the repeated-filtering configuration optically, apart from the efficient realization of the desired system, we also have to take into account the limitations associated with the practical realization of several optical fractional Fourier filters. The cumulative effects of diffraction, scattering, and attenuation will ultimately limit the possible number of stages. It is for this reason that we have emphasized systems involving only moderate numbers of stages in our numerical examples, although from an algorithmic viewpoint the method is applicable for larger values of $M$ as well. In a digital implementation of the repeated-filtering configuration, we do not have such restrictions.

The cost of the system, either digital or optical, increases with the number of stages. On the other hand, a greater number of filters allows a better approximation to the desired linear system or matrix. This is the basic performance-cost trade-off in repeated filtering. The plots in Figs. 4 and 5 can also be interpreted as typical performance-cost trade-off figures. We can choose the most attractive performance-cost point on the curve by selecting the number of stages appropriately. For instance, the intrinsic accuracy of analog optical systems is limited to approximately a dynamic range of 100 or so. Given this accuracy, it is pointless to try to implement the desired linear system by using a scheme that could in principle accommodate much greater accuracies (such as a conventional matrix-vector-multiplier architecture). Furthermore, when we are implementing digital optical interconnection architectures, even greater inaccuracies can be tolerated while still maintaining an acceptable eye pattern. Thus the proposed method allows one to reduce the cost of implementing such systems.

Naturally, the number of filters required to attain a given accuracy will be smaller for matrices or tensors exhibiting greater regularity or more subtle forms of intrinsic structure. The regularity or the structure inherent in a matrix may be exploited on a case-by-case basis through ingenuity or invention. Most fast algorithms are obtained in this manner. In contrast, our method provides a systematic way of obtaining an efficient implementation that does not require ingenuity on a case-by-case basis. This approach would be especially useful when the regularity or the structure of the matrix is not expressed symbolically or when we are presented with a specific matrix in numerical form that does not have any evident regularity or structure.

\section{CONCLUSION}

In this paper we have formulated the problem of approximating linear systems by using repeated filtering in consecutive domains. The utility of the method is illustrated by several examples.

In the first example we restore signals degraded by space-variant turbulence by synthesizing the corresponding optimum linear estimator with our repeated-filtering method. In the second example we propose an efficient way of generating the moments of signals. In the third one we synthesize the Hadamard transformation, and, finally, in the fourth example we consider the implementation of optical permutation architectures.

In all of the examples, we saw that we could obtain useful approximations of the desired linear transformations with our repeated-filtering configuration by using a moderate number of filters. The cost of implementing these systems (optically or digitally) is much less than the cost of implementing general linear systems. We also saw that excellent approximations are also possible with phase-only filters.

The basic method proposed can be applied to many different applications where one seeks to efficiently realize a linear system or a matrix-vector product. If the matrix or the tensor at hand has some kind of intrinsic structure, we may obtain better approximations with a smaller number of filters. In such cases computing the matrixvector product digitally in $O\left(N^{2}\right)$ time or realizing it with a conventional matrix-vector-product architecture requiring the space-bandwidth product $N^{2}$ is clearly inefficient. This is one case where the proposed repeatedfiltering method would be useful, allowing one to exploit the intrinsic structure as much as possible in a systematic manner.

A distinct circumstance in which the method may be beneficial, even when such an intrinsic structure does not exist, is that in which we wish to compute the matrixvector product or realize the linear system with limited accuracy. This may be the case when some other component or stage of the overall system limits the accuracy to a lower value anyway, when we are transmitting digital signals, or simply when the application demands limited accuracy.

If the approximation obtained with a given number of filters is not sufficient, we must increase the number of filters to obtain a better approximation. Thus the proposed method allows us to trade off efficiency and accuracy.

\section{REFERENCES}

1. H. M. Ozaktas, B. Barshan, D. Mendlovic, and L. Onural, "Convolution, filtering, and multiplexing in fractional Fourier domains and their relation to chirp and wavelet transforms," J. Opt. Soc. Am. A 11, 547-559 (1994).

2. M. A. Kutay, H. M. Ozaktas, L. Onural, and O. Arikan, “Optimal filtering in fractional Fourier domains," in Proceedings of the International Conference on Acoustics, Speech, 
and Signal Processing (IEEE, Piscataway, N.J., 1995), pp. 937-940.

3. M. A. Kutay, H. M. Ozaktas, O. Arikan, and L. Onural, "Optimal filtering in fractional Fourier domains," IEEE Trans. Signal Process. 45, 1129-1143 (1997).

4. M. F. Erden, H. M. Ozaktas, and D. Mendlovic, "Synthesis of mutual intensity distributions using the fractional Fourier transform," Opt. Commun. 125, 288-301 (1996).

5. H. M. Ozaktas, O. Arikan, M. A. Kutay, and G. Bozdagi, "Digital computation of the fractional Fourier transform," IEEE Trans. Signal Process. 44, 2141-2150 (1996).

6. H. M. Ozaktas and D. Mendlovic, "Fourier transforms of fractional order and their optical interpretation," Opt. Commun. 101, 163-169 (1993).

7. A. W. Lohmann, "Image rotation, Wigner rotation, and the fractional order Fourier transform," J. Opt. Soc. Am. A 10, 2181-2186 (1993).

8. H. M. Ozaktas and D. Mendlovic, "Fractional Fourier optics," J. Opt. Soc. Am. A 12, 743-751 (1995).

9. P. Pellat-Finet and G. Bonnet, "Fractional order Fourier transform and Fourier optics," Opt. Commun. 111, 141-154 (1994).

10. M. F. Erden, M. A. Kutay, and H. M. Ozaktas, "Repeated filtering in consecutive fractional Fourier domains and its application to signal restoration," IEEE Trans. Signal Process. (to be published).

11. J. W. Goodman, A. R. Dias, and L. M. Woody, "Fully parallel, high-speed incoherent optical method for performing discrete Fourier transforms," Opt. Lett. 2, 1-3 (1978).

12. S. K. Case, P. R. Haugen, and O. J. Løberge, "Multifacet holographic optical elements for wave front transformations," Appl. Opt. 20, 2670-2675 (1981).

13. H. M. Ozaktas and D. Mendlovic, "Multistage optical interconnection architectures with the least possible growth of system size,” Opt. Lett. 18, 296-298 (1993).

14. A. C. McBride and F. H. Kerr, "On Namias's fractional Fourier transform," IMA J. Appl. Math. 39, 159-175 (1987).

15. D. Mendlovic and H. M. Ozaktas, "Fractional Fourier transforms and their optical implementation. I," J. Opt. Soc. Am. A 10, 1875-1881 (1993).

16. H. M. Ozaktas and D. Mendlovic, "Fractional Fourier transforms and their optical implementation. II," J. Opt. Soc. Am. A 10, 2522-2531 (1993).

17. L. B. Almeida, "The fractional Fourier transform and timefrequency representations," IEEE Trans. Signal Process. 42, 3084-3091 (1994).

18. H. M. Ozaktas and M. F. Erden, "Relationships among ray optical, Gaussian beam, and fractional Fourier transform descriptions of first-order optical systems," Opt. Commun. 143, 75-86 (1997).

19. B. E. A. Saleh and M. C. Teich, Fundamentals of Photonics (Wiley, New York, 1991).

20. M. J. Bastiaans, "Wigner distribution function and its application to first-order optics," J. Opt. Soc. Am. 69, 17101716 (1979)

21. K. B. Wolf, Integral Transforms in Science and Engineering (Plenum, New York, 1979).

22. M. Nazarathy and J. Shamir, "First-order optics-a canonical operator representation: lossless systems," J. Opt. Soc. Am. 72, 356-364 (1982).

23. S. Abe and J. T. Sheridan, "Optical operations on wave functions as the Abelian subgroups of the special affine Fourier transformation," Opt. Lett. 19, 1801-1803 (1994).

24. A. Sahin, H. M. Ozaktas, and D. Mendlovic, "Optical implementation of the two-dimensional fractional Fourier transform with different orders in the two dimensions," Opt. Commun. 120, 134-138 (1995).

25. M. F. Erden, H. M. Ozaktas, A. Sahin, and D. Mendlovic, "Design of dynamically adjustable fractional Fourier transformer," Opt. Commun. 136, 52-60 (1997).

26. L. M. Bernardo and O. D. D. Soares, "Fractional Fourier transforms and imaging," J. Opt. Soc. Am. A 11, 2622-2626 (1994).

27. T. Alieva, V. Lopez, F. Agullo-Lopez, and L. B. Almedia, "The fractional Fourier transform in optical propagation problems," J. Mod. Opt. 41, 1037-1044 (1994).

28. D. Mendlovic, Z. Zalevsky, A. W. Lohmann, and R. G. Dorsch, "Signal spatial-filtering using the localized fractional Fourier transform," Opt. Commun. 126, 14-18 (1996).

29. J. Garcia, D. Mendlovic, Z. Zalevsky, and A. Lohmann, "Space-variant simultaneous detection of several objects by the use of multiple anamorphic fractional-Fouriertransform filters," Appl. Opt. 35, 3945-3952 (1996).

30. D. Mendlovic, H. M. Ozaktas, and A. W. Lohmann, "Fractional correlation," Appl. Opt. 34, 303-309 (1995).

31. A. W. Lohmann, Z. Zalevsky, and D. Mendlovic, "Synthesis of pattern recognition filters for fractional Fourier processing," Opt. Commun. 128, 199-204 (1996).

32. J. C. Wood and D. T. Barry, "Linear signal synthesis using the Radon-Wigner transform," IEEE Trans. Signal Process. 42, 2105-2111 (1994).

33. Z. Zalevsky and D. Mendlovic, "Fractional Wiener filter," Appl. Opt. 35, 3930-3936 (1996).

34. D. F. McAlister, M. Beck, L. Clarke, A. Mayer, and M. G. Raymer, "Optical phase retrieval by phase-space tomography and fractional Fourier transforms," Opt. Lett. 20, 1181-1183 (1995)

35. B. Barshan, M. A. Kutay, and H. M. Ozaktas, "Optimal filtering with linear canonical transformations," Opt. Commun. 135, 32-36 (1997).

36. A. Sahin, H. M. Ozaktas, and D. Mendlovic, "Optical implementations of two-dimensional fractional Fourier transforms and linear canonical transforms with arbitrary parameters," Appl. Opt. 37, 2130-2141 (1997).

37. A. Sahin, "Two-dimensional fractional Fourier transform and its optical implementation," M.S. thesis (Bilkent University, Department of Electrical and Electronics Engineering, Ankara, Turkey, 1996).

38. H. M. Ozaktas, "Repeated fractional Fourier domain filtering is equivalent to repeated time and frequency domain filtering," Signal Process. 54, 81-84 (1996).

39. H. M. Ozaktas and D. Mendlovic, "Every Fourier optical system is equivalent to consecutive fractional Fourier domain filtering," Appl. Opt. 35, 3167-3170 (1996).

40. A. K. Jain, Fundamentals of Digital Image Processing (Prentice-Hall, Englewood Cliffs, N.J., 1989).

41. J. L. Horner, Optical Signal Processing (Academic, London, 1987).

42. D. Mendlovic and H. M. Ozaktas, "Optical-coordinate transformation methods and optical-interconnection architectures," Appl. Opt. 32, 5119-5124 (1993). 